\title{
Qualidade física e química da água em sistema fechado de recirculação durante o cultivo de juvenis de tilápia-do-Nilo
}

\section{Physical and chemical quality of water in closed recirculation system during the cultivation of Nile tilapia juveniles}

\author{
Nilton Garcia Marengoni ${ }^{1 *}$; Francisca Lílian Sales Mota ${ }^{2}$; \\ Raimundo Bemvindo Gomes ${ }^{3}$; Francisca Francineuma Fernandes Basílio4; \\ Newton Tavares Escocard de Oliveira ${ }^{5}$; Masayoshi Ogawa ${ }^{6}$
}

\begin{abstract}
Resumo
O trabalho teve por objetivo avaliar o efeito do filtro e do tempo de cultivo de alevinos de tilápia-doNilo sobre a qualidade de água do sistema de recirculação fechado. Foram utilizados 1.350 alevinos de tilápia-do-Nilo (Oreochromis niloticus) em delineamento experimental de blocos casualizados e esquema de parcelas subdivididas no tempo, com doze tratamentos e três repetições. Os tratamentos foram constituídos pela combinação de dois locais de coleta de água (antes do filtro mecânico e após o filtro biológico) e de seis tempos de cultivo (14, 28, 42, 56, 70 e 84 dias), em esquema de parcelas subdivididas. A temperatura, oxigênio dissolvido, saturação de oxigênio, condutividade elétrica e pH não afetaram a desenvolvimento dos peixes em sistema fechado de recirculação de água. Observou-se que a água coletada após o filtro biológico apresentou melhor qualidade física e química que a coletada antes do filtro mecânico. Os níveis de oxigênio dissolvido na água após o filtro biológico foram maiores $(\mathrm{P}<0,05)$ aos observados antes do filtro mecânico e houve redução linear gradativa das concentrações de oxigênio dissolvido em função dos dias de cultivo. Com relação aos parâmetros sólidos totais, sólidos suspensos totais, amônia total e nitrato observou-se que a redução na qualidade da água do sistema foi mais evidente $(\mathrm{P}<0,05)$ no período de 56 a 84 dias.

Palavras-chave: Oreochromis niloticus, filtros biológicos e mecânicos, qualidade de água
\end{abstract}

\begin{abstract}
The study aimed to evaluate the effect of the filter and time of cultivation of Nile tilapia juveniles in water quality in closed recirculating system. There were used 1,350 fingerlings of Nile tilapia (Oreochromis niloticus) in a randomized block experimental design and split-plot time with twelve treatments and three replicates. The treatments were a combination of two local of water harvesting (before the mechanical filter and after the biological filter) and six periods of cultivation (14, 28, 42, 56, 70 and 84 days), in a split plot arrangement. The water temperature, dissolved oxygen, oxygen saturation, electrical conductivity and $\mathrm{pH}$ did not affect the development of the fish in closed recirculating water. It was
\end{abstract}

\footnotetext{
${ }^{1}$ Prof. do Centro de Ciências Agrárias da Universidade Estadual do Oeste do Paraná, UNIOESTE, Marechal Cândido Rondon, PR. E-mail:nmarengoni@hotmail.com

2 M.e. em Recursos Pesqueiros e Engenharia de Pesca, Universidade Federal do Ceará, UFC, Fortaleza, CE. E-mail: lilianpesca@, yahoo.com.br

3 Prof. do Instituto Federal de Educação, Ciência e Tecnologia, IFCE, Fortaleza, CE. E-mail: rgomes@cefet.br

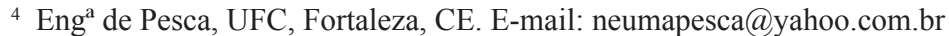

${ }^{5}$ Prof. do Centro de Ciências Agrárias, UNIOESTE, Marechal Cândido Rondon, PR. E-mail: newtonescocard@hotmail.com

${ }^{6}$ Dr. em Recursos Pesqueiros e Engenharia de Pesca, UFC, Fortaleza, CE. E-mail: ogawa@ufc.br

* Autor para correspondência
} 
observed that the water collected after the biological filter had better physical and chemical quality than that collected before the mechanical filter. The dissolved oxygen levels in the water after the biological filter were higher $(\mathrm{P}<0.05)$ to those observed before the mechanical filter and there was a gradual linear reduction of dissolved oxygen concentrations in terms of days of cultivation. Concerning the values of total solids, total suspended solids, total ammonia and nitrate was observed that the reduction in water quality of the system was more evident $(\mathrm{P}<0.05)$ within 56 to 84 days.

Key words: Oreochromis niloticus, biological and mechanical filters, water quality

Os peixes cultivados dependem, entre muitos fatores, da qualidade de água e de rações nutricionalmente completas que garantam o desempenho produtivo e a sanidade. Além do desempenho, o potencial poluente das rações deve fazer parte das preocupações de piscicultores e fabricantes, visando minimizar a carga de agentes poluentes lançados no ambiente.

Atualmente, busca-se a prática da aquicultura responsável, visando à produção lucrativa de organismos aquáticos, concomitantemente com a não degradação do meio ambiente, e com a geração de benefícios sociais. A aquicultura moderna envolve os componentes essenciais e indissociáveis para que a atividade seja perene (OSTRENSKY; BOEGER, 2008).

A qualidade da água em aquicultura é o conjunto de características ótimas que devem ser mantidas no ambiente, garantindo o sucesso dos cultivos (ARANA, 2004). Esse conjunto se caracteriza pelo equilíbrio dinâmico de todas as variáveis físicas, químicas, biológicas e tecnológicas, que torna possível o cultivo sustentável de organismos aquáticos, atendendo aos objetivos sociais, ambientais e econômicos da realidade conjuntural em que o empreendimento encontra-se inserido.

Ridha e Cruz (2001) caracterizam o sistema fechado de recirculação de água como uma produção em que água flui pela unidade de cultivo e atravessa um filtro, que pode ser mecânico, no qual será removido o material particulado, tais como, resíduos de ração, fezes e sólidos e, biológico que possuirá a função de purificar a água que circula constantemente promovendo a transformação do nitrogênio amoniacal, nitrato ou $\mathrm{N}_{2}$.
Carvalho (2005) aborda que o sistema de recirculação de água foi desenvolvido e aprimorado para locais em que possuem limitações quanto à captação de água evitando assim ao máximo o desperdício do recurso hidrológico existente em áreas onde a água é escassa ou de má qualidade, aumentando a produção por volume de água ao longo de todo ano, além de controlar a entrada de patógenos. Os sistemas de recirculação também podem ser integrados a outros cultivos animais ou vegetais, melhorando a eficiência produtiva da atividade aquícola sem que haja prejuízo ao meio ambiente (ALI et al., 2010).

O trabalho teve por objetivo avaliar o efeito do filtro e do tempo de cultivo de juvenis de tilápia-doNilo (Oreochromis niloticus) na qualidade de água do sistema de recirculação fechado, submetidos à dieta contendo alto valor proteico e energético.

O estudo foi conduzido no Laboratório de Recursos Aquáticos (LARAq) da Universidade Federal do Ceará (UFC), no período de abril a julho de 2008. Utilizaram-se 1.350 alevinos de tilápia com peso médio inicial de $0,6 \pm 0,12 \mathrm{~g}$ e comprimento total inicial de $3,15 \pm 0,22 \mathrm{~cm}$, em delineamento experimental de blocos casualizados completos e esquema de parcelas subdivididas no tempo, com doze tratamentos e três repetições. Os peixes foram uniformemente distribuídos em 27 tanquesrede alocados nas nove caixas dos três sistemas de recirculação.

Os 12 tratamentos foram constituídos pela combinação de dois locais de coleta de água (antes do filtro mecânico e após o filtro biológico do sistema de recirculação) e de seis tempos de cultivo $(14,28$, $42,56,70$ e 84 dias). Os locais caracterizaram as 
parcelas e os tempos constituíram as subparcelas. $\mathrm{O}$ bloco foi constituído de três caixas com três tanquesrede cada, um filtro mecânico e um filtro biológico, representando um sistema fechado de recirculação de água. A utilização de blocos teve a finalidade de controlar a variação de composição química e física do ambiente aquático entre os sistemas.

Utilizaram-se nove caixas de $1.000 \mathrm{~L}$, distribuídas em três sistemas independentes em que foram fixados três tanques-rede de $0,1 \mathrm{~m}^{3}$ em cada caixa. A água do sistema recirculava, com auxílio de bomba de sucção submersível (modelo Sarlo Better) com capacidade de vazão de $2 \mathrm{~m}^{3} \mathrm{~h}^{-1}$, do filtro biológico, composto por conchas de ostras, para cada uma das caixas de cultivo e destas, por gravidade, para o filtro mecânico que continha camadas de brita que retinham as partículas em suspensão. Não houve renovação da água e o volume dos sistemas manteve-se constante com a reposição apenas da água perdida por evaporação.

Os peixes foram alimentados quatro vezes ao dia e os comedouros utilizados eram sifonados para a retirada das fezes e sobras de ração experimental peletizada $(2 \mathrm{~mm})$, contendo $12 \%$ de farelo da amêndoa da castanha de caju entre outros ingredientes, caracterizando uma dieta de alto valor proteico (42\% PB) e energético (4.528 kcal kg-1). $\mathrm{Na}$ fase inicial do cultivo os peletes foram moídos e peneirados para atender o tamanho dos alevinos.-

As variáveis avaliadas foram: temperatura, condutividade, oxigênio dissolvido, saturação de oxigênio, demanda bioquímica de oxigênio (DBO), fósforo total (P-total), $\mathrm{pH}$, sólidos totais (ST), sólidos suspensos totais (SST), amônia total (AT), nitrogênio total (NTK) e nitrato total (NIT) no 14을 $28^{\circ}, 42^{\circ}, 56^{\circ}, 70^{\circ}$ e $84^{\circ}$ dia de cultivo das tilápias, antes do filtro mecânico e após o filtro biológico do sistema de recirculação de água.

A salinidade da água do sistema de recirculação foi monitorada por refratômetro modelo RTS101ATC e mantida em $0,2 \%$ ao longo do período com a adição de sal na água durante as biometrias.
$\mathrm{O} \mathrm{pH}$, a condutividade, o oxigênio dissolvido, a temperatura e a saturação de $\mathrm{O}_{2} \mathrm{D}$ da água foram mensurados diariamente, no início da manhã e ao final da tarde, com o auxílio de aparelho digital multiparâmetro modelo YSI 556.

Nas biometrias quinzenais foram coletadas seis amostras compostas, a partir de quatro subamostras de água ao longo do dia, no ponto de entrada na caixa de cultivo (após o filtro biológico) e na saída da caixa de cultivo (antes do filtro mecânico) para cada um dos três sistemas de recirculação. As subamostras foram coletadas em béquer de $2000 \mathrm{~mL}$ e estocadas em seis baldes previamente esterilizados com capacidade de 10 L. Posteriormente, os baldes foram lacrados e mantidos sob refrigeração. Ao final do dia, as amostras de água foram acondicionadas dentro de recipiente térmico e transportadas ao Laboratório Integrado de Águas Mananciais e Residuárias (Liamar), situado no Instituto Federal de Educação, Ciência e Tecnologia (IFCE), Fortaleza, $\mathrm{CE}$, onde foram submetidas às análises de sólidos totais, sólidos suspensos totais, DBO, amônia total, nitrogênio total, nitrato total e fósforo total, segundo a metodologia descrita por APHA (1999).

A normalidade dos erros experimentais e a homogeneidade de variâncias entre os tratamentos para as diversas variáveis foram avaliadas previamente utilizando-se os testes de Shapiro-Wilk e de Levene, respectivamente. Os resultados indicaram que os erros experimentais de temperatura, condutividade, oxigênio dissolvido, saturação e DBO apresentaram distribuição normal de probabilidades e homogeneidade de variâncias dos tratamentos.

A variável fosforo total (PT) foi transformada para logaritmo do fósforo total (LOGPT), para atender aos pressupostos de normalidade e homogeneidade de variâncias dos erros.

Para a temperatura, condutividade, oxigênio dissolvido (OD), saturação de oxigênio, DBO e LOGPT, o modelo estatístico utilizado foi: $\mathrm{Y}_{\mathrm{ijk}}=$ $\mathrm{m}+\mathrm{L}_{\mathrm{i}}+\mathrm{B}_{\mathrm{k}}+\boldsymbol{\varepsilon}_{\mathrm{ik}}+\mathrm{T}_{\mathrm{j}}+\mathrm{LT}_{\mathrm{ij}}+\varepsilon_{\mathrm{ijk}}$, em que: $\mathrm{Y}_{\mathrm{ijk}}=$ 
observação média de cada subparcela na classe i, no nível j de cada parcela e no bloco $\mathrm{k} ; \mathrm{m}=$ efeito da média geral do experimento; $\mathrm{L}_{\mathrm{i}}=$ efeito de local de coleta de água, para $\mathrm{i}=(1,2) ; \mathrm{B}_{\mathrm{k}}=$ efeito dos blocos, para $\mathrm{k}=(1,2,3) ; \varepsilon_{\mathrm{ik}}=$ erro aleatório da parcela medido na classe i e no bloco $\mathrm{k} ; \mathrm{T}_{\mathrm{j}}=$ efeito de tempo de cultivo, para $\mathrm{j}=(1,2,3,4,5,6) ; \mathrm{LT}_{\mathrm{ij}}$ $=$ efeito da interação entre local e tempo; $\varepsilon_{\mathrm{ijk}}=$ erro aleatório associado à subparcela, medido na classe $\mathrm{i}$, no nível j e no bloco $\mathrm{k}$.

Os efeitos de local e de tempo de cultivo na temperatura, condutividade, OD, saturação de oxigênio, DBO e LOGPT foram verificados pela análise de variância (ANOVA). O efeito de local foi comparado por meio do teste $F$ na ANOVA e o efeito de tempo sobre as variáveis foi estimado por equações polinomiais, respeitando-se os resultados significativos obtidos na análise de variância da regressão.

Para as variáveis $\mathrm{pH}$, sólidos totais (ST), sólidos suspensos totais (SST), amônia total (AT), nitrogênio total (NTK) e nitrato total (NIT) foi adotada uma estratégia não-paramétrica, em que se utilizou o teste de Friedman para avaliar a hipótese de nulidade conjunta de que todas as somas de ranks $\left(\sum \mathrm{R}_{\mathrm{i}}\right)$ dos tratamentos são equivalentes $\left(\mathrm{H}_{0}: \sum \mathrm{R}_{1}=\right.$ $\left.\sum \mathrm{R}_{2}=\ldots=\sum \mathrm{R}_{12}\right)$. Posteriormente, foi utilizado $\mathrm{o}$ teste de Wilcoxon para teste dos escores obtidos nos períodos de 14 a 42 versus 56 a 84 dias, em cada local de coleta de água.

O nível de significância de 0,05 foi adotado em todos os testes estatísticos. As análises foram realizadas utilizando-se o programa Statistical Analyses System (SAS, 2000).

Houve efeito $(\mathrm{P}<0,05)$ de interação entre local de coleta da água e tempo de cultivo das tilápias na temperatura da água. Os maiores valores de temperaturas da água foram obtidos antes do filtro mecânico, quando a água saia das caixas de cultivo, com exceção para a coleta realizada no $70^{\circ}$ dia de cultivo.
Os valores médios de temperatura variaram de 25,20 a $26,90^{\circ} \mathrm{C}$ e estão em conformidade para o cultivo de tilápia-do-Nilo segundo Boyd e Tucker (1998), e mantendo-se próximos da faixa de tolerância de conforto térmico recomendada para a criação de tilápia, que varia entre 27 a $32^{\circ} \mathrm{C}$ (EL-SAYED, 2006). Em sistemas de recirculação El-Sayed e Kawanna (2008) demonstraram que a temperatura ótima de conforto térmico para tilápia é $28^{\circ} \mathrm{C}$.

Houve efeito $(\mathrm{P}<0,05)$ polinomial de tempo de cultivo na condutividade $(\mathrm{y}=-2,81+0,30 \mathrm{x}$ $\left.-0,0016 \mathrm{x}^{2} ; \mathrm{R}^{2}=0,91\right)$ e demanda bioquímica de oxigênio $\left(\mathrm{y}=-187,56+14,109 \mathrm{x}-0,1348 \mathrm{x}^{2} ; \mathrm{R}^{2}=\right.$ $0,76)$ e efeito linear sobre o oxigênio dissolvido (y $\left.=6,97-0,06 x ; R^{2}=0,85\right)$, saturação de oxigênio $\left(y=86,405-0,7519 x ; R^{2}=0,85\right)$ e logaritmo do fósforo total $\left(\mathrm{y}=-0,56+0,0166 \mathrm{x} ; \mathrm{R}^{2}=0,97\right)$, independentemente do local de coleta da água.

O aumento na condutividade ao longo do período de cultivo pode ser explicado pelo acúmulo de íons oriundos da mineralização dos resíduos de ração, das excretas dos peixes (RAFIEE; SAAD, 2005) e do manejo profilático com aplicação de sal à água do cultivo durante as biometrias. Segundo Lima (2005), níveis superiores a $100 \mu \mathrm{S} \mathrm{cm}^{-1}$ indicam ambientes impactados. No entanto, os índices observados do início ao final das coletas mantiveram-se próximos aos valores adequados para a tilapicultura intensiva (EL-SAYED, 2006).

O local de coleta da água influenciou $(\mathrm{P}<0,05)$ na concentração de oxigênio dissolvido (OD) e na saturação de oxigênio (SAT), independentemente do tempo de cultivo. Os valores médios de OD (4,50 \pm $\left.1,66 \mathrm{mg} \mathrm{L}^{-1}\right)$ e SAT $(55,30 \pm 20,15 \%)$ foram maiores $(\mathrm{P}<0,05)$ na água coletada após o filtro biológico, quando estava retornando às caixas de cultivo do sistema, em comparação aos valores de OD (3,50 $\left.\pm 1,70 \mathrm{mg} \mathrm{L}^{-1}\right)$ e SAT $(43,83 \pm 22,65 \%)$ na água coletada antes do filtro mecânico o que demonstrou eficiência no funcionamento dos filtros. Porém, ao longo do cultivo os níveis de OD chegaram próximos 
a $2 \mathrm{mg} \mathrm{L}^{-1}$ que são críticos para a tilapicultura intensiva, quando o sistema atingiu a capacidade de suporte e o experimento foi finalizado (Figura 1). A alta concentração dos compostos nitrogenados pode ter influenciado na dinâmica do oxigênio dissolvido no meio (PEREIRA; MERCANTE, 2005) e consequentemente condições inadequadas como a redução dos níveis de OD e acúmulo de matéria orgânica podem ter contribuído na redução da eficiência dos filtros.

Figura 1. Valores médios de oxigênio dissolvido (O2D) e fósforo total (P-total) encontrado na água dos sistemas de recirculação em função do local de coleta e de dias de cultivo dos juvenis de tilápia.

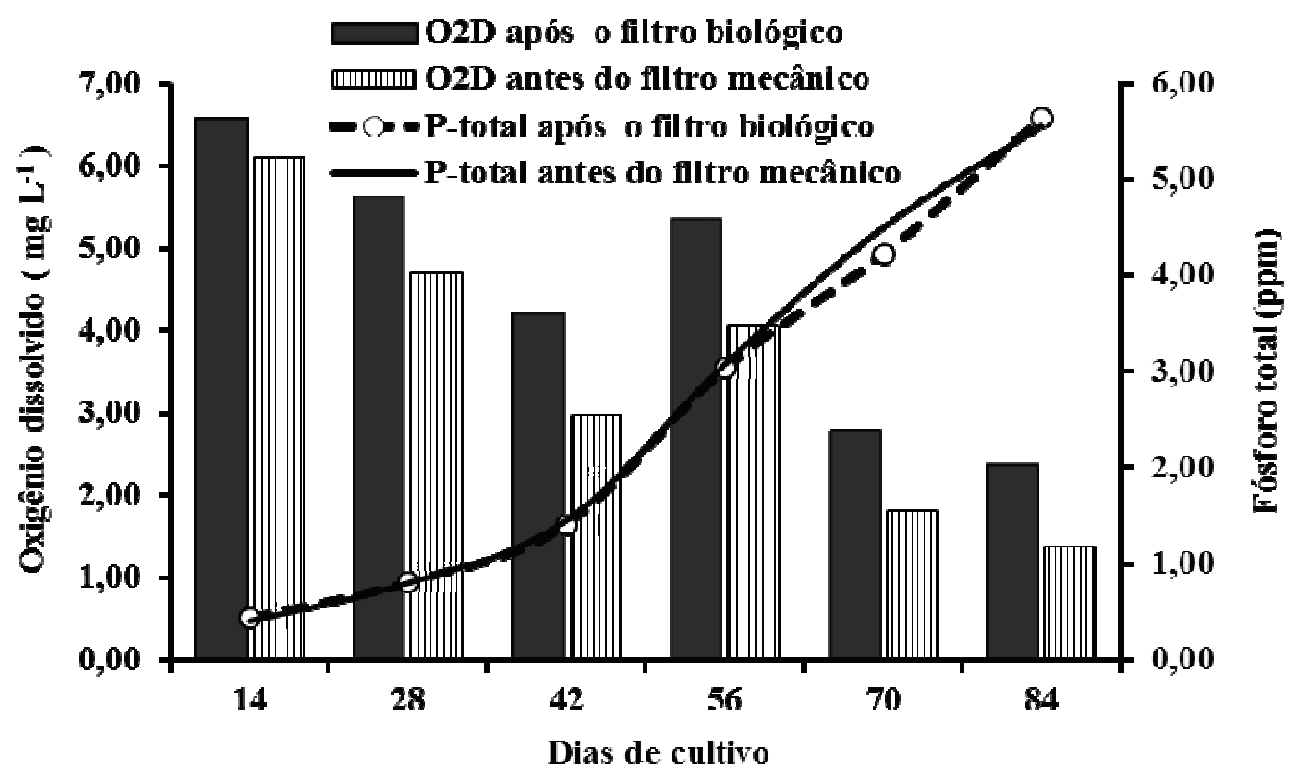

Fonte: Elaboração dos autores.

Observou-se que a taxa de redução do oxigênio dissolvido foi de $0,06 \mathrm{mg} \mathrm{L}^{-1}$ para cada dia de cultivo intensivo de tilápia no sistema de recirculação fechado, porém para a água coleta após o filtro biológico, os valores mínimos foram de $2,4 \mathrm{mg}$ $\mathrm{L}^{-1}$ no final do cultivo (Figura 1). A redução linear gradativa das concentrações de oxigênio dissolvido em função dos dias de cultivo pode ser atribuída ao aumento da quantidade de ração fornecida aos peixes, ao aumento da produção de dejetos e a decomposição de matéria orgânica. Os fatores que causam a redução do oxigênio dissolvido incluem a velocidade de degradação da matéria orgânica, os excedentes alimentícios, a presença de fezes, o incremento da taxa metabólica devido ao aumento da temperatura (oscilações noitedia), a desgaseificação do oxigênio da água para a atmosfera, a densidade do cultivo e a redução da agitação da água que ocasiona a diminuição da superfície de troca (ESPAÑA, 2008).

Os níveis de fósforo total aumentaram em função dos dias de cultivo (Figura 1). Isso pode ter sido influenciado pela liberação de compostos fosfatados provenientes da decomposição da matéria orgânica de sobras de ração e da excreção metabólica. Uma pequena taxa de fósforo que entra no sistema aquático via ração é retido na biomassa dos peixes (CYRINO et al., 2010), sendo que os resíduos das dietas podem causar a deterioração da qualidade da água e baixas econômicas (ALI et al., 2010). Para 
tilápias o limite de tolerância que pode oscilar entre 0,6 e 1,5 ppm de fósforo total (ESPAÑA, 2008), sendo que este limite foi atingido após o $42^{\circ}$ dia de cultivo.

Verifica-se na Tabela 1 que houve efeito $(\mathrm{P}<0,05)$ dos tratamentos sobre o $\mathrm{pH}$, sólidos totais (ST), sólidos suspensos totais (SST), amônia total (AT) e nitrogênio total (NTK). Não foi possível detectar diferença significativa $(\mathrm{P}=0,2124)$ dos tratamentos sobre o teor de nitrato total (NIT). A concentração de amônia total e nitrogênio total mantiveram-se abaixo de 2 ppm até o $56^{\circ}$ dia de cultivo.

A toxidez do nitrato em animais aquáticos parece não ser um sério problema, porém, este composto torna-se potencialmente tóxico em sistemas de recirculação de água, em que altos níveis podem ser alcançados como resultado da nitrificação da amônia. A toxidez deste composto é devido a seu efeito sobre a osmorregulação e possivelmente sobre o transporte de oxigênio (ARANA, 2004). Neste trabalho a sobrevivência média foi de $80 \%$ e os possíveis efeitos negativos dos compostos nitrogenados sobre o metabolismos dos peixes podem ter sido minimizados pela correlação dos efeitos benéficos de outras variáveis físicas e químicas, especialmente da temperatura, $\mathrm{pH}$, condutividade da água que mantiveram adequadas (EL-SAYED, 2006) até o $42^{\circ}$ dia de cultivo dos juvenis de tilápia. Outro aspecto positivo pode ter sido atribuído à manutenção da salinidade da água a $0,2 \%$, que além de não interferir no funcionamento do filtro biológico, contribui na prevenção de intoxicação por nitrito.

Tabela 1. Escores médios para pH, sólidos totais (ST), sólidos suspensos totais (SST), amônia total (AT), nitrogênio total (NTK) e nitrato total (NIT) nos períodos de 14 a 42 e de 56 a 84 dias em cada local de coleta de água do sistema de recirculação, e estatística calculada e nível descritivo.

\begin{tabular}{|c|c|c|c|c|c|c|c|}
\hline \multirow{2}{*}{ Local no sistema } & \multirow{2}{*}{ Tempo (dias) } & \multicolumn{6}{|c|}{ Característica } \\
\hline & & $\mathrm{pH}$ & ST & SST & AT & NTK & NIT \\
\hline \multirow[t]{2}{*}{ Após o filtro biológico } & $(14,28$ e 42$)$ & 7,53 & 2140,11 & 8,22 & 0,35 & 1,34 & 3,05 \\
\hline & $(56,70$ e 84$)$ & 7,47 & 6135,00 & 33,78 & 7,27 & 11,47 & 2,77 \\
\hline \multirow{2}{*}{\multicolumn{2}{|c|}{ 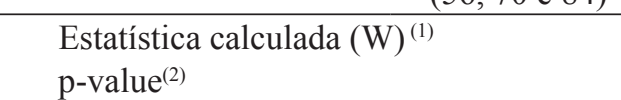 }} & $-4,0$ & 22,5 & 21,5 & 22,5 & 22,5 & 1,5 \\
\hline & & 0,1875 & 0,0019 & 0,0039 & 0,0019 & 0,0019 & 0,4551 \\
\hline \multirow[t]{2}{*}{ Antes do filtro mecânico } & $(14,28$ e 42$)$ & 7,44 & 2205,67 & 8,11 & 0,45 & 1,42 & 3,11 \\
\hline & $(56,70$ e 84$)$ & 7,33 & 6035,78 & 43,67 & 7,48 & 12,70 & 2,24 \\
\hline \multirow{2}{*}{\multicolumn{2}{|c|}{ 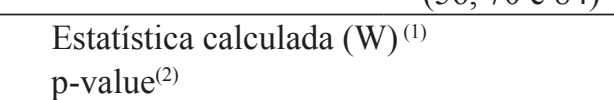 }} & $-10,5$ & 22,5 & 22,5 & 22,5 & 22,5 & 6,5 \\
\hline & & 0,1250 & 0,0019 & 0,0019 & 0,0019 & 0,0019 & 0,2481 \\
\hline \multirow{2}{*}{\multicolumn{2}{|c|}{$\begin{array}{l}\chi^{2(3)} \\
p-\text { value }^{(4)}\end{array}$}} & 27,1563 & 30,9207 & 30,5024 & 32,3326 & 31,7935 & 14,3846 \\
\hline & & $0,0044 *$ & $0,0011^{*}$ & $0,0013 *$ & $0,0007 *$ & $0,0008^{*}$ & $0,2124^{\text {ns }}$ \\
\hline
\end{tabular}

(1) Comparação não-paramétrica da soma dos postos, em valor absoluto, menos o valor médio $(\mathrm{n}(\mathrm{n}+1) / 4)$, para $\mathrm{n}=$ número de pares sem empates; ${ }^{(2)}$ Probabilidade de significância pelo teste dos postos sinalizados de Wilcoxon, com hipótese alternativa unilateral à direita.

(3)- $\chi_{\text {cal }}^{2}=12 \times[b t(t+1)]^{-1} \times\left\lfloor\sum_{i=1}^{1} r_{i}^{2}-3 b(t+1)\right\rfloor$, em que: $b=3=n^{0}$ de blocos; $t=12=n^{o}$ de tratamentos (combinação de 2 locais no tanque x 6 tempos de medição); $r_{i}=$ total das b repetições no tratamento $i$; obtido de 36 observações; $H_{0}=$ os tratamentos são

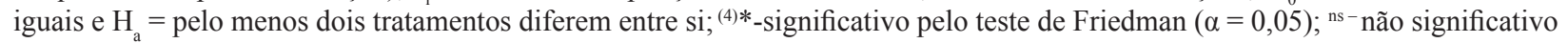
$(\mathrm{P}>0,05)$

Fonte: Elaboração dos autores. 
A comparação não-paramétrica dos escores médios de $\mathrm{pH}$, sólidos totais (ST), sólidos suspensos totais (SST), amônia total (AT), nitrogênio total (NTK) e nitrato total (NIT) nos períodos de 14 a 42 versus 56 a 84 dias, em cada local de coleta da água, encontra-se na Tabela 1. Observou-se que a redução na qualidade da água do sistema foi mais evidente a partir do $56^{\circ}$ dia de cultivo, com maiores $(\mathrm{P}<0,05)$ teores de ST, SST, AT e NTK no período de 56 a 84 dias, tanto para a água coletada antes do filtro mecânico quanto após o filtro biológico. Esperavase que a partir da estabilização do filtro biológico ao longo do cultivo, ocorresse uma diminuição dos níveis de amônia total e possivelmente um aumento nos teores de nitrato devido à ação das bactérias no processo de nitrificação da amônia (PEREIRA; MERCANTE, 2005).

Os valores médios de sólidos totais (ST), que inicialmente encontravam-se elevados, e sólidos suspensos totais (SST) ficaram acima do tolerado pelos peixes a partir do décimo quarto dia de coleta e aumentaram gradativamente (BRASIL, 2005). A quantidade e a digestibilidade da dieta utilizada com a inclusão de $12 \%$ de farelo da amêndoa da castanha do caju (FACC) pode ter contribuído para o impacto dos sólidos no sistema fechado de recirculação de água. Possivelmente o elevado teor proteico e energético da dieta e a liberação de resíduos poluidores da água contribuíram para a neutralização dos efeitos positivos dos filtros, especialmente a partir do $56^{\circ}$ dia e estendendo-se até período final do cultivo de juvenis no sistema fechado de recirculação de água.

Os valores médios de $\mathrm{pH}$ observados mantiveram-se próximos aos recomendados para o cultivo de peixes. $\mathrm{O} \mathrm{pH}$ da água é muito importante para a aquicultura porque interfere no metabolismo e nos processos fisiológicos dos peixes. Os pontos letais de acidez e alcalinidade são de $\mathrm{pH}=4$ e pH $=11$, respectivamente. As águas com valores que compreendem a faixa de 6,5 a 9,0 são as mais adequadas para a produção de peixes (BOYD; TUCKER, 1998; BRASIL, 2005).

Os níveis de amônia total aumentaram com o tempo de cultivo, apresentando maiores valores a partir do 56을 dia, tanto na entrada como na saída dos filtros (Tabela 1). A faixa de amônia na água deve ser mantida entre 0,02 a $0,1 \mathrm{mg} \mathrm{L}^{-1}$ (valores próximos a $2 \mathrm{mg} \mathrm{L}^{-1}$ são críticos), pois se houver uma concentração alta de amônia na água surge uma série de consequências negativas, como o bloqueio do metabolismo, lesões em brânquias e órgãos internos, alteração no balanço de sais, imunossupressão e vulnerabilidade à doenças e redução na taxa de crescimento e sobrevivência (BOYD; TUCKER, 1998; BRASIL, 2005).

Os níveis de nitrogênio total foram menores até o $42^{\circ}$ dia, após o filtro biológico e antes do filtro mecânico do sistema de recirculação. Assim como o fósforo total o nitrogênio residual da aquicultura merece atenção, pois estes efluentes podem causar a eutrofização dos sistemas aquáticos, caso não seja respeitado o limite da capacidade de carga dos ecossistemas aquáticos, bem como a capacidade de reciclagem dos nutrientes (RAFIEE; SAAD, 2005; CYRINO et al., 2010).

A qualidade física e química da água é melhor na saída do filtro biológico que na entrada do filtro mecânico instalados em sistemas fechados de recirculação de água durante o cultivo de juvenis de tilápia-do-Nilo. A partir do $56^{\circ}$ dia, os níveis dos compostos nitrogenados aumentam e a eficiência do filtro biológico diminui, influenciando na qualidade da água do sistema.

\section{Agradecimentos}

À Fundação Cearense de Apoio ao Desenvolvimento Científico e Tecnológico (FUNCAP), pelo auxílio financeiro à pesquisa. 


\section{Referências}

ALI, M.; HAYWARD, R. S.;BAJER, P.G.;WHITLEDGE, G. W. Maintenance/submaximum feeding schedules for reducing solid wastes and improving feed conversion in aquaculture. Journal of the World Aquaculture Society, Hoboken, v. 41, n. 3, p. 319-331, 2010.

AMERICAN PUBLIC HEALTH ASSOCIATION APHA. Standard methods for the examination of water and wastewater. $20^{\text {th }}$ ed. New York: American Public Health Association, 1999. 1325 p.

ARANA, L. V. Princípios químicos da qualidade da água em aquicultura. 2. ed. Florianópolis: UFSC, 2004. $231 \mathrm{p}$.

BOYD, C. E.; TUCKER, C. S. Pond aquaculture water quality management. Boston: Kluwer Academic Publishers, 1998. 700 p.

BRASIL. CONAMA - CONSELHO NACIONAL DO MEIO AMBIENTE. Normas, padrões de qualidade e classificação das águas. Resolução $\mathrm{n}^{\circ} 357$, de 17 de março de 2005. Diário Oficial [da] República Federativa do Brasil, Brasília, 18 mar. 2005, Seção 1. p. 58-63.

CARVALHO, M. B. Larvicultura de beijupirá. Panorama da Aquicultura, Rio de Janeiro, v. 15, n. 92, p. 45-53, 2005.

CYRINO, J. E. P.; BICUDO, A. J. A.; SADO, R. Y.; BORGHESI, R.; DAIRIKI, J. K. A piscicultura e o ambiente: o uso de alimentos ambientalmente corretos em piscicultura. Revista Brasileira de Zootecnia, Viçosa, v. 39, p. 68-87, 2010. Suplemento.

EL-SAYED, A. F. M. Tilapia culture. London: CABI publishing, 2006. 277 p.

EL-SAYED, A. F. M.; KAWANNA, M. Optimum water temperature boosts the growth performance of Nile tilapia (Oreochromis niloticus) fry reared in a recycling system. Aquaculture Research, Hoboken, v. 39, n. 6, p. 670-672, 2008.
ESPAÑA. Ministério da Agricultura. Pesca y Alimentación. Factibilidad del cultivo de Tilapia em Mallorca. 2008. Disponível em: <http://wwwmapa. pesca/publicaciones/ publi_recientes.htm>. Acesso em: 10 jan. 2012.

LIMA, M. A. S. Águas acumuladas em açudes e barragens na região de Santa Maria e flutuações em seus atributos físico químicos. 2005. Dissertação (Mestrado em Ciência do Solo) - Universidade Federal de Santa Maria, Santa Maria.

OSTRENSKY, A.; BOEGER, W. A. Principais problemas enfrentados atualmente pela aquicultura brasileira. In: OSTRENSKY, A.; BORGUETTI, J. R.; SOTO, D. Aquicultura no Brasil: o desafio é crescer. Brasília: SEAP/FAO, 2008. p. 135-158.

PEREIRA, L. P. F.; MERCANTE, C. T. J. A amônia nos sistemas de criação de peixes e seus efeitos sobre a qualidade da água. Uma revisão. Boletim do Instituto de Pesca, São Paulo, v. 31, n. 1, p. 81-88, 2005.

RAFIEE, G.; SAAD, C. R. Nutrient cycle and sludge production during different stages of red tilapia (Oreochromis sp.) growth in a recirculating aquaculture system. Aquaculture, Amsterdam, v. 244, n. 1-4, p. 109118, 2005.

RIDHA, M. T.; CRUZ, E. M. Effect of biofilter media on water quality and biological performance of the Nile tilapia Oreochromis niloticus L. reared in a simple recirculation system. Aquaculture Engeneering, Seattle, v. 24 , n. 2, p. 157-166, 2001.

STATISTICAL ANALYSIS SYSTEM INSTITUTE SAS. User's guide. Version 8.2. ${ }^{\text {th }}$. v. 2. Cary: 2000. 